\title{
Clinical Characteristics of the Forehead Lipoma
}

\author{
Jong-Seo Lee, \\ So-Min Hwang, \\ Yong-Hui Jung, \\ Hong-ll Kim, \\ Hyung-Do Kim, \\ Min-Kyu Hwang, \\ Min-Wook Kim
}

Aesthetic, Plastic and Reconstructive Surgery Center, Good Moonhwa Hospital, Busan, Korea

No potential conflict of interest relevant to this article was reported.

\begin{abstract}
Background: Lipomas can be categorized into deep and superficial lipomas according to anatomical depth. Many cases of forehead lipomas are reported to be deep to the muscle layer. We analyze ultrasound in delineating depth of forehead lipomas.

Methods: A retrospective review was performed for all patients who underwent excision of forehead lipomas between January 2008 and March 2013 and for whom preoperative ultrasound study was available. Sensitivity and specificity of ultrasound imaging was evalauted against depth finding at the time of surgical excision.

Results: The review identified 42 patients who met the inclusion criteria. Preoperative ultrasound reading was 18 as deep lipomas and 24 as superficial. However, intraoperative finding revealed 2 of the 18 deep lipomas to be superficial and 13 of the 24 superficial lipomas to be deep lipomas. Overall, ultrasonography turned out to be $69 \%(29 / 42)$ accurate in correctly delineating superficial versus deep lipomas.

Conclusion: Lipomas of the forehead tend to be located in deeper tissue plane compared to lipomas found elsewhere in the body. Preoperative ultrasonography of lipomas can be helpful, but was not accurate in identifying the depth of forehead lipomas in our patient population. Even if a forehead lipoma is found to be superficial on ultrasound, operative planning should include the possibility of deep lipomas.
\end{abstract}

Keywords: Lipoma / Forehead / Ultrasonography

\section{INTRODUCTION}

A lipoma is the most common benign connective tissue tumor. Histologically, it is surrounded by thin connective tissue membrane and its inner consists of normal adipocytes [1]. Depending on its relationship to the muscle fascia, a lipoma can be classified as superficial or deep [2]. In the forehead, deep lipomas have been reported at a higher incidence, when compared to other locations in the body [3]. Surgical approach and planning for the deep lipoma differ from those for the superficial lipoma, and therefore, ac-

Correspondence: So-Min Hwang

Aesthetic, Plastic and Reconstructive Surgery Center, Good Moonhwa Hospital, 199 Beomil-ro, Dong-gu, Busan601-803, Korea

E-mail: sominhwang@hanmail.net

Received September 24, 2014 / Revised November 23, 2014 /

Accepted December 5, 2014 curate preoperative diagnosis is important. Although ultrasonography is widely used as a tool of preoperative diagnosis, a possibility of misdiagnosis in determining the accurate location of lipomas exists [4]. Accordingly, we aimed to investigate the ratio of deep lipomas in the forehead and the accuracy of ultrasonography.

\section{METHODS}

A retrospective review was performed for patients with histologically diagnosed forehead lipomas, among those who had visited our hospital with soft tissue mass as a chief complaint. Preoperative diagnosis was made with ultrasonography by radiologists at our institution. The ultrasonography equipment used was Phillips IU-22 (Bothwell, WA, USA). 


\section{RESULTS}

The review identified 42 cases of forehead lipoma between March 2008 and August 2013. Among these, 27 were deep lipo$\mathrm{ma}$, and 15 were superficial lipoma. The number of patients diagnosed with deep lipoma in preoperative ultrasonography was 18 , and 16 of them were consistently diagnosed with the deep lipoma postoperatively. Twenty-four patients were diagnosed with the superficial lipoma in preoperative ultrasonography. However, only 13 of these 24 patients were confirmed to have the superficial lipoma with the remaining 11 as having deep lipoma (Table 1). Over-

Table 1. Preoperative ultrasonographic findings and results of biopsy

\begin{tabular}{lccc}
\multirow{2}{*}{ Results of biopsy } & \multicolumn{2}{c}{ Preoperative ultrasonographic findings } & \\
\cline { 2 - 3 } Deep & Deep & Superficial & \\
\hline Deep & 16 & 11 & 27 \\
Superficial & 2 & 13 & 15 \\
Total & 18 & 24 & 42 \\
\hline
\end{tabular}
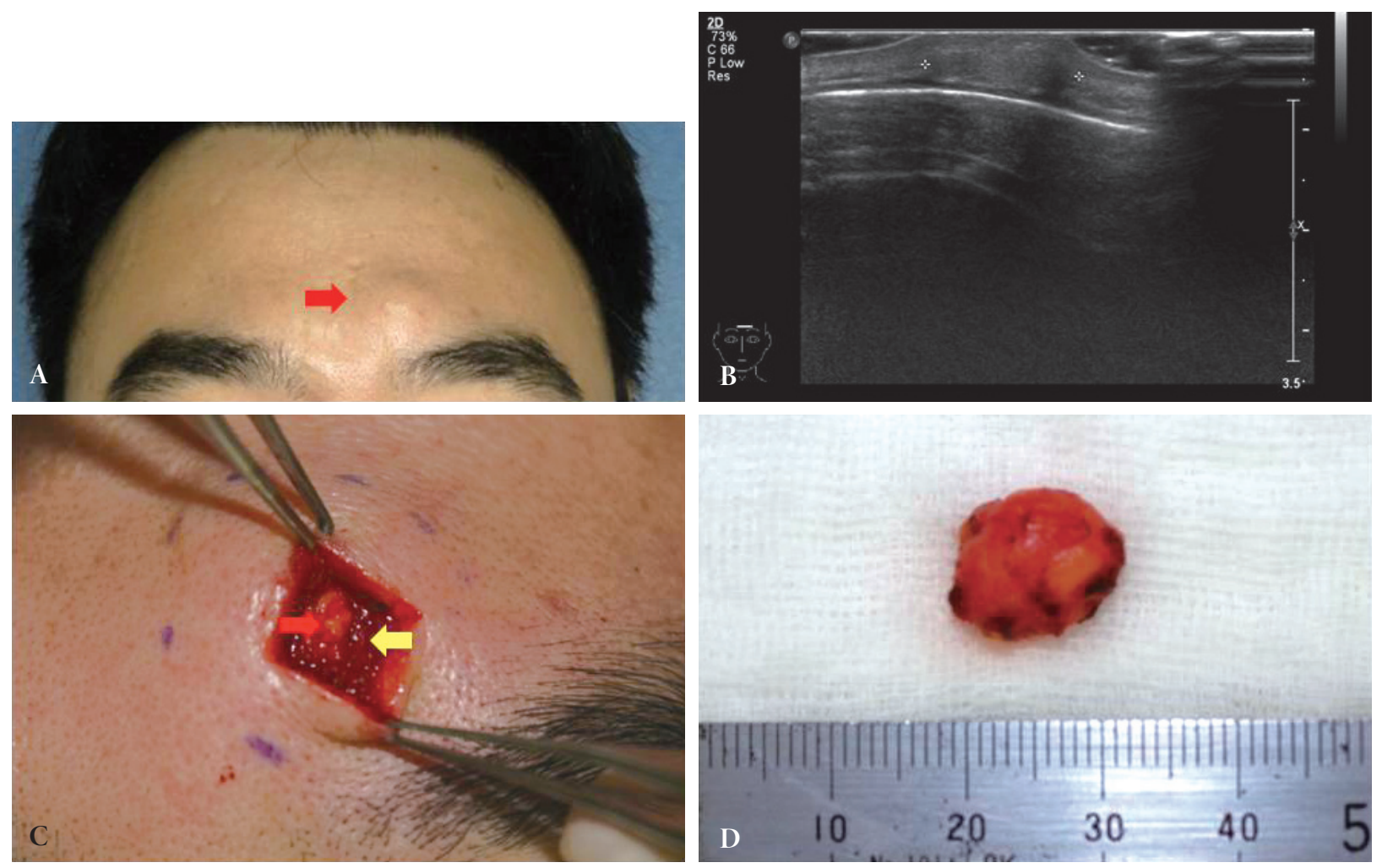

all, the accuracy of ultrasonography on forehead lipoma depth was $69 \%(29 / 42)$.

\section{Case}

\section{Case 1}

A 36-year-old male patient visited our hospital with a palpable mass on the forehead as a chief complaint. Preoperative ultrasonography suggested the mass to be in the subcutaneous layer, and the impression was that of a superficial lipoma. During the operation, however, the mass was found to be deep to the frontalis muscle and required a deeper plane of dissection prior to excision. The mass measured $1.5 \times 1.3 \times 1.2 \mathrm{~cm}$ and was confirmed to be a lipoma on histologic examination (Fig. 1).

\section{Case 2}

A 55-year-old female patient presented with a palpable fore-

Fig. 1. Case 1. (A) A protruding mass was placed on forehead (red arrow). (B) The mass was diagnosed as superficial lipoma in sonography. (C) The mass (red arrow) is located beneath muscle (yellow arrow) and diagnosed as deep lipoma intraoperatively. (D) The size of the mass was $1.5 \times$ $1.3 \times 1.2 \mathrm{~cm}$ and finally diagnosed as a lipoma in biopsy. 
head mass. Preoperative ultrasound suggested a superficial lipoma. As with the patient in case 1 , the lipoma was located deep to the muscle plane and required a more extensive dissection (Fig. 2).

\section{DISCUSSION}

A lipoma is a benign form of tumor composed of mature white adipocytes, and the most common soft tissue mesenchymoma in adults [1]. Depending on the depth in relation to muscle fascia, lipomas can be classified into superficial and deep lipomas, with the latter including intramuscular and submuscular lipomas [2]. Deep lipomas account for $60 \%$ of all forehead lipomas [5], which was $64.2 \%$ in our study. This is significantly higher than relative ratio of deep lipomas occuring in other locations $(0.4 \%-5.7 \%)$ $[6,7]$. This higher proportion of deep lipomas in the forehead could be attributed to anatomic characteristics of the forehead. Salasche et al. [8] suggested that forehead skin can glide well since superficial musculo-aponeurotic system and periosteum were divided by the loose areola tissue in subgaleal space. They suggested such anatomical relationship is what allows traumatic scalp avulsion and is also why lipomas exist in the deeper plane. In addition, studies by Tamura et al. [3] and Zitelli [9] suggested that subgaleal lipomas were heterotopic lipomas that did not occur from adipose tissue but from other mesenchymal tissues, which subsequently could cause lipomas in the subgaleal area.

Because of availability and low cost, ultrasonography has been widely used in the diagnosis of lipomas [4]. However, the ultrasonography may be inaccurate in delineating lipoma depth. Since the forehead lipomas are soft, they can be compressed by the ultrasound probe and taken on a morphology in parallel with skin and muscle fascia [10]. In addition, tissue layers of the forehead can become thinner from mass effect of lipomas, which contributes to the difficulty in elucidating lipoma depth. Therefore, a surgeon should be careful when making operative plans according to
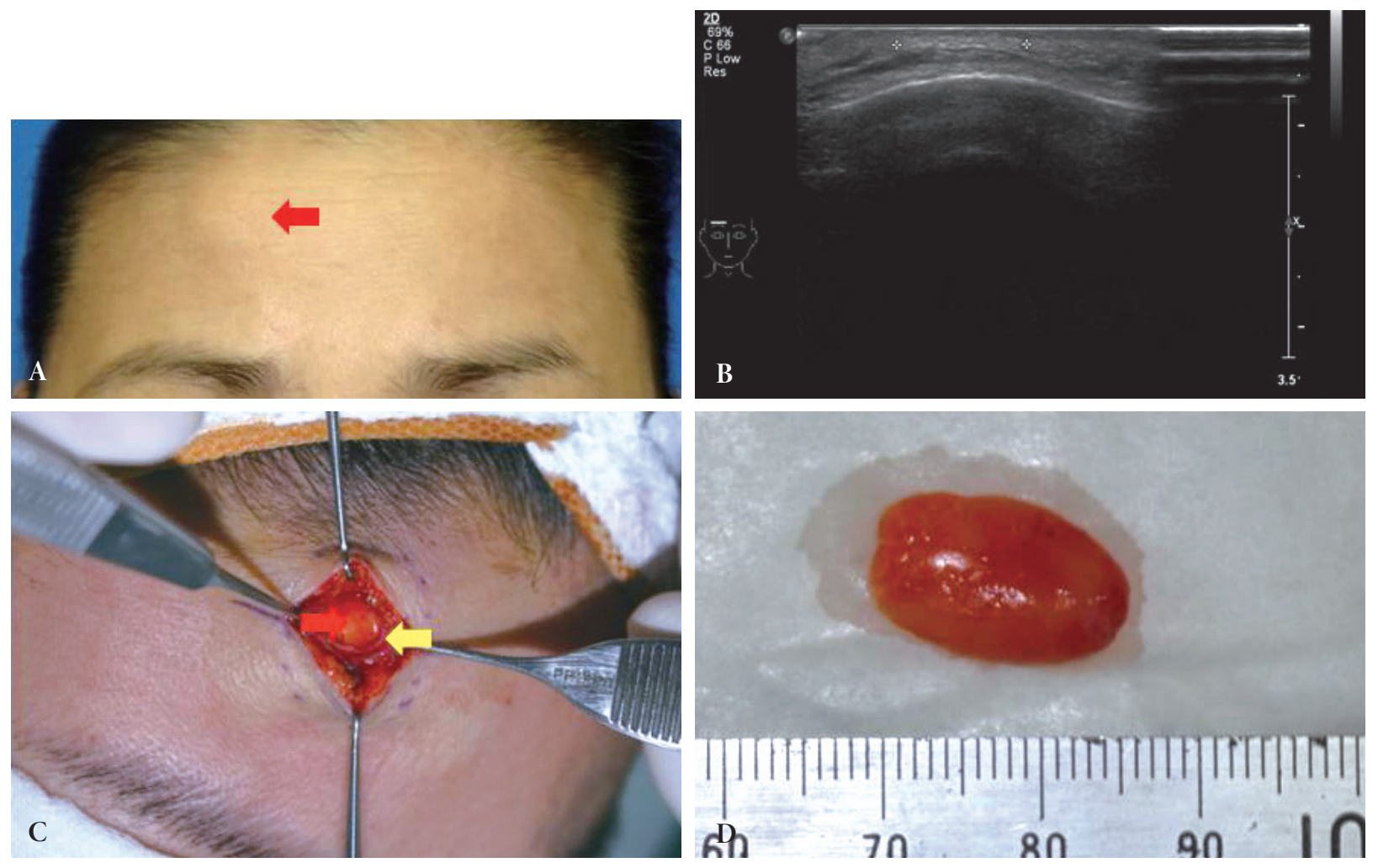

Fig. 2. Case 2. (A) A protruding mass was placed on forehead (Red arrow). (B) The mass was diagnosed as superficial lipoma in sonography. (C) The mass (red arrow) is located beneath muscle (yellow arrow) and diagnosed as deep lipoma intraoperatively. (D) The size of the mass was $1.5 \times$ $1.0 \times 0.9 \mathrm{~cm}$ and finally diagnosed as a lipoma in biopsy. 
preoperative diagnosis from ultrasound imaging.

In this retrospective review, lipomas were classified according to the depth relative to muscle fascia. A limitation of our study is that the deep lipomas were not further classified into intramuscular, submuscular, or subgaleal lipomas.

Forehead lipomas are likely to be located in the deep plane, and preoperative diagnosis is important since more care must be taken to avoid major structural damage during the excision of such lipomas located in the deep plane. Even if preoperative imaging reveals a forehead lipoma to be superficial, operations should be planned with the possibility of the lipoma actually being in the deeper plane since ultrasonography has a fairly low accuracy in determining the depth of forehead lipomas.

\section{REFERENCES}

1. Fletcher CD, Unni KK, Mertens F. World Health Organization; International Agency for Research on Cancer. Pathology and genetics of tumours of soft tissue and bone. Lyon: IARC Press; 2002.
2. Weiss SW, Goldblum JR, Enzinger FM. Enzinger and Weiss's soft tissue tumors. 5th ed. St. Louis: Elsevier; 2008.

3. Tamura A, Ishikawa O, Miyachi Y. Subgaleal angiomyolipoma. J Dermatol 1994;21:514-7.

4. Inampudi P, Jacobson JA, Fessell DP, Carlos RC, Patel SV, DelaneySathy LO, van Holsbeeck MT. Soft-tissue lipomas: accuracy of sonography in diagnosis with pathologic correlation. Radiology 2004;233: 763-7.

5. Grosshans E, Fersing J, Marescaux J. Subaponeurotic lipoma of the forehead. Ann Dermatol Venereol 1987;114:335-40.

6. Myhre-Jensen O. A consecutive 7-year series of 1331 benign soft tissue tumours. Clinicopathologic data. Comparison with sarcomas. Acta Orthop Scand 1981;52:287-93.

7. Rydholm A, Berg NO. Size, site and clinical incidence of lipoma. Factors in the differential diagnosis of lipoma and sarcoma. Acta Orthop Scand 1983;54:929-34.

8. Salasche SJ, McCollough ML, Angeloni VL, Grabski WJ. Frontalisassociated lipoma of the forehead. J Am Acad Dermatol 1989;20:4628.

9. Zitelli JA. Subgaleal lipomas. Arch Dermatol 1989;125:384-5.

10. Ahuja AT, King AD, Kew J, King W, Metreweli C. Head and neck lipomas: sonographic appearance. AJNR Am J Neuroradiol 1998;19: 505-8. 